Peter Philippson

In der Welt träumen

11. Jahrgang, Nr. 2, 2021, Seite 33-39

DOI: 10.30820/1664-9583-2021-2-33

Psychosozial-Verlag

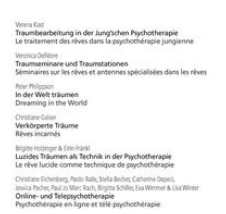




\section{Impressum}

Psychotherapie-Wissenschaft

ISSN 1664-9583 (Print-Version)

ISSN 1664-9591 (digitale Version)

11. Jahrgang Heft $2 / 2021$

https://doi.org/10.30820/1664-9583-2021-2

info@psychotherapie-wissenschaft.info

www.psychotherapie-wissenschaft.info

\section{Herausgeber}

Schweizer Charta für Psychotherapie in der Assoziation

Schweizer Psychotherapeutinnen und Psychotherapeuten

Geschäftsstelle ASP

Riedtlistr. 8

CH-8006 Zürich

Tel. +41432689300

www.psychotherapie.ch

\section{Redaktion}

Mara Foppoli, Lugano

Lea-Sophie Richter, Zürich

Mario Schlegel, Zürich

Peter Schulthess, Zürich

Hinweise für AutorInnen befinden sich auf der Homepage der Zeitschrift: www.psychotherapie-wissenschaft.info

\section{Verlag}

Psychosozial-Verlag

Walltorstr. 10

D-35390 Gießen

+49642196997826

info@psychosozial-verlag.de

www.psychosozial-verlag.de

\author{
Abo-Verwaltung \\ Psychosozial-Verlag \\ bestellung@psychosozial-verlag.de
}

\section{Bezugsgebühren}

Jahresabonnement 44,90€ (zzgl. Versand)

Einzelheft $24,90 €$ (zzgl. Versand)

Studierende erhalten gegen Nachweis $25 \%$ Rabatt.

Das Abonnement verlängert sich um jeweils ein Jahr, sofern nicht eine Abbestellung bis acht Wochen vor Ende des Bezugszeitraums erfolgt.

ASP-Mitglieder wenden sich wegen des Abonnements bitte direkt an die ASP.

\section{Anzeigen}

Anfragen zu Anzeigen bitte an den Verlag:

anzeigen@psychosozial-verlag.de

Es gelten die Preise der auf www.psychosozial-verlag.de einsehbaren Mediadaten.

ASP-Mitglieder wenden sich bitte direkt an

die ASP-Geschäftsstelle: asp@psychotherapie.ch

\section{Digitale Version}

Die Zeitschrift Psychotherapie-Wissenschaft ist auch online einsehbar: www.psychotherapie-wissenschaft.info

\section{(C) $(1) \Theta$}

Die Beiträge dieser Zeitschrift sind unter der Creative Commons Attribution-NonCommercial-NoDerivs 3.0 DE Lizenz lizensiert. Diese Lizenz erlaubt die private Nutzung und unveränderte Weitergabe, verbietet jedoch die Bearbeitung und kommerzielle Nutzung. Weitere Informationen finden Sie unter: creativecommons.org/licenses/by-nc-nd/3.0/de 


\title{
In der Welt träumen
}

\author{
Peter Philippson \\ Psychotherapie-Wissenschaft 11 (2) 2021 33-39 \\ www.psychotherapie-wissenschaft.info \\ CC BY-NC-ND \\ https://doi.org/10.30820/1664-9583-2021-2-33
}

\begin{abstract}
Zusammenfassung: Meiner Ansicht nach sind Träume in vielerlei Hinsicht sehr wichtig. Hier sehe ich eine ähnliche Signifikanz wie Sigmund Freud und Fritz Perls. Regelmässig bringe ich die Arbeit mit Träumen in meine Therapien, Schulungen und Gruppenarbeit ein, damit unsere Wahrnehmung auf das gerichtet wird, was auf individueller und auf kollektiver Ebene geschieht. Mein Artikel ist in zwei Teile gegliedert. Der erste Teil ist eine Übersicht, wie ich Träume sehe und auf welche unterschiedlichen Arten ich mit ihnen als Therapeut gearbeitet habe. Im zweiten Teil stelle ich eine Arbeitsweise mit Träumen vor, die ich in den letzten Jahren entwickelt habe. Dabei wird sowohl das Verständnis wie die Arbeit in eine andere Richtung gelenkt, in der die strikte Trennung zwischen dem Wachsein und Träumen aufgehoben ist.
\end{abstract}

Schlüsselwörter: Traum, Kunstwerke, Feldprozesse, Interpretation, Wachträume, Träume als Realität, Paare

\section{Was ist ein Traum?}

Ich schlage vor, dass wir Träume primär als Kunstwerke sehen, als improvisierte postmoderne Theaterstücke, die wir geschrieben haben, bei denen wir Regie führen, in denen wir die Figuren spielen und bei denen wir auch in erster Linie die Zuschauenden sind! Unsere Träume umfassen Liebesgeschichten, Horror und Spannung, absurdes Theater, Alltägliches, Krieg und die Sphären, die als spirituell oder transpersonal betrachtet werden können. Wir können sogar eine Reihe verwandter Kunstwerke produzieren, bei denen der Subtext von einem Werk zum nächsten mitgenommen wird. Auch diejenigen, die ansonsten diese Art Theaterstücke ablehnen, sind produktive Schöpfende in diesem Genre, obwohl sie wahrscheinlich auch ihre Träume ablehnen würden. Ein Traum sagt uns, dass wir im Kern kreative, künstlerische Wesen sind.

Wenn wir Träume als Kunst sehen, macht uns das hoffentlich skeptisch gegenüber einer vereinfachenden oder einschränkenden Suche nach Bedeutung, gleichsam als ginge es bei Romeo und Julia um die Gefahren eines schlechten Postwesens. Wie Stanislavsky sagte, geht es im Theater um den Subtext - den Text kann man auch zu Hause lesen. Wenn wir also mit Träumen arbeiten, den eigenen oder denen von Klient*innen, müssen wir damit beginnen, dass wir dem Subtext Platz bieten und uns von ihm überschwemmen lassen, bevor wir irgendetwas anderes mit ihm anstellen. Bringen Sie der Kunst keinen Respekt entgegen, wird der*die Künstler*in Sie nicht weiter teilhaben lassen. Traumarbeit ist langsam und verlangt nach Kreativität; und nicht hauptsächlich nach einer Interpretation durch die linke Gehirnhälfte. Bei der Auslegung gibt es auch immer das grosse Problem der kulturellen Voreingenommenheit, wenn angenommen wird, dass Bedeutungen allgemeingültig sind, sodass diverse kulturelle Abweichungen in Bezug auf die Signifikanz von Symbolen und Bildern übersehen werden.
Zum Beispiel können Träume vom Tod für Menschen, die an ein Leben nach dem Tod glauben, eine ganz andere Bedeutung haben als für diejenigen, die das nicht tun, und dann noch einmal für diejenigen, die glauben, dass ihr Leben nach dem Tod glücklich (himmlisch) sein wird, und diejenigen, die den Tod zum Fürchten (höllisch) finden.

Die Motivation zum Träumen ist - in guter Gestaltpsychologie/Therapietradition - üblicherweise etwas, das unfertig ist und nach weiterem Ausdruck sucht, wenn zensierende Aktivitäten unseres normalen wachen Lebens im Schlaf oder beim Dösen entfallen. Und für die zwei Hauptversionen dieser unfertigen Situation gelten die zwei Hauptansätze der Gestalttherapie für das Arbeiten mit Träumen.

\section{Träume als Projektionen}

Das klassische Konzept des «unfinished business» der Gestalttheorie, nach dem Grundsatz von Prägnanz oder des Zeigarnik-Effekts (Zeigarnik, 1927), ist ein offensichtlicher Anwärter dafür, was unser Träumen motiviert (es besagt, dass die Unterbrechung einer Aufgabe die Chancen erhöht, dass wir uns an sie erinnern). Ein wesentlicher Prozess, der nach Vollendung strebt, wird im Traum ausgelebt und die Charaktere sowie das Umfeld des Traums können als Projektionen der Möglichkeiten behandelt werden, die eine Vollendung gestatten könnten. Auf diese Weise arbeitete Fritz Perls hauptsächlich mit Träumen. Er bat Klient*innen als die Figuren des Traums zu sprechen (nicht nur als Person oder lebende Figur, sondern auch als Objekt, als der Traum selbst oder als das, was darin fehlte - unsichtbar oder überaschenderweise nicht vorkommend). Die Interaktionen dieser Charaktere, wie sie einander unterstützen oder sich gegenseitig bekämpfen, können ebenfalls erforscht werden, sodass ein besseres Gefühl für die im Traum implizierten Möglichkeiten entsteht. 


\section{Beispiel}

Klient: Ich träumte, ich sei in mein Elternhaus gegangen und allein darin herumgewandert, dabei fühlte ich dessen vertraute, wenn auch leicht unangenehme Stimmung. Dann sah ich eine Tür, die ich nie zuvor gesehen hatte. Sie war klein und man konnte sie leicht übersehen, aber ich wollte wissen, was sich dahinter verbirgt. Ich musste mich bücken, um hindurchgehen zu können. Ich fand mich in einem hellen Raum mit grossen Fenstern wieder. Es waren Leute darin, die verschiedene Spiele spielten. Ich wusste nicht, ob ich mich ihnen anschliessen sollte, obwohl sie einladend wirkten.

Therapeut: Können Sie als die Tür sprechen? Was ist der Traum aus ihrer Perspektive?

Klient: Ich war schon immer da, aber ich bin klein und so wurde ich nicht bemerkt. Man muss sich kleiner machen, wenn man durch mich gehen will, ohne sich den Kopf zu stossen.

Therapeut: Wie würden Sie als die Tür die Unterschiede zwischen der einen und der anderen Seite beschreiben?

Klient: Auf der einen Seite ist da dieser Mann, der allein herumwandert und nur beobachtet. Auf der anderen Seite sind Leute, Aktivitäten, Energie. Ich bin überrascht, dass der Mann sie nicht gehört hat, ich bin nicht besonders schalldicht.

Von dieser Art der Projektionsarbeit kann der Klient mit den Themen Isolation, dem Nicht-Wahrnehmen von Möglichkeiten, sich mit dem Leben zu verbinden, und dem Konzept, dass er sich kleiner machen muss, um einen neuen Raum betreten zu können, in Kontakt kommen.

\section{Träume als Retroflektionen}

Der andere, von Isadore From bevorzugte Ansatz, beginnt mit dem Konzept, dass unser Träumen nicht grundsätzlich von der Welt abgetrennt, sondern der Anfang eines Prozesses ist, der in einer Aktion endet, mit der der Traum verwirklicht wird. Ich kann von einem Gebäude träumen und es dann Wirklichkeit werden lassen, ich kann von einer Beziehung träumen, zum Beispiel einer sexuellen, und jemanden bitten, dazuzukommen und den Traum mit mir in der Realität umzusetzen. Dies ist die Gestalttheorie der Aggression, gemäss der Menschen nicht passiv in eine Welt eintreten, sondern willentlich, und die Welt gleichzeitig mit dem Betreten nach ihrem Willen neu gestalten.

Bei Froms Herangehensweise ist ein Traum eine $R e$ troflektion dieses Vollendungsprozesses in der Welt, als träumte man, wenn man hungrig ist vom Essen, anstatt sich Essen zu besorgen. From betrachtete Träume aus der Nacht vor einer Therapiesitzung als retroflektierte Aussagen, die sich an Therapeut*innen richten, und bat Klient*innen, Wege zu finden, diese Aussage nach aussen zu kehren. Meiner Ansicht nach ist dies eine etwas beschränkte, wenn auch mitunter sinnvolle Anwendung eines fruchtbaren
Konzepts, bei dem das künstlerische Schaffen in einer ziemlich banalen Kommunikation verloren gehen kann, so wie ein Klient From bekanntermassen einen Traum mitbrachte, den er als «kurz» bezeichnete, und From erforschte die Beziehung des Klienten zu seiner «kleinen» Statur. Aber die Bedeutung hinter dem, was die Realisierung des Traumes in der Welt wäre, ist ein Ansatz, der mich fasziniert, und das Sich-durch-einen-Traum-Arbeiten kann dann vielmehr in einer Aktion als nur mit Worten stattfinden. Es werden damit auch andere Möglichkeiten für das Arbeiten mit dem Traum eröffnet, sodass man auch damit spielen kann, dem Traum einen anderen Ausgang zu geben, und sich dann ansieht, was passiert.

Mit demselben Traum könnte die Therapie wie folgt weitergehen:

Therapeut: Ich habe eine Idee. Sie könnten damit experimentieren, dass Sie diesen Raum durch die Tür verlassen und ich bleibe hier. Dann kommen Sie wieder herein und wir können sehen, was dann passiert.

Klient: [verlässt den Raum, bleibt ein paar Minuten draussen und klopft dann an der Tür]

Therapeut: [sitzt, wirft einen Ball und fängt ihn wieder]

Klient: [klopft erneut und tritt schliesslich ein] Ich habe darauf gewartet, dass Sie mir sagen, ich könne hereinkommen. [verunsichert und leicht verärgert]

Therapeut: [spielt weiter mit dem Ball, lächelt den Klienten dabei an und tut dann so, als werfe er ihm den Ball zu]

Klient: [fängt den Ball und hält ihn fest, schaut dabei noch immer verunsichert]

Therapeut: Wenn Sie also durch die Tür hinausgehen, haben Sie damit Ihr Recht verloren, im Raum zu sein; die Tür, durch die Sie so viele Male gekommen sind, ist eine neue Tür geworden und eine Schranke. Für Sie scheint es nun schwierig, die Frage «Möchte ich mit Ihnen spielen?» zu beantworten. Und meine Frage ist «Welche Türöffnung lassen Sie mich sein?»

Mit dieser Art des Arbeitens werden die Traumbilder als Retroflektionen der Beziehung zwischen Klient und Therapeut gesehen; wem gehört der Raum, bin ich willkommen, möchte ich spielen, kann ich mir erlauben, die neuen Räume zu sehen, zu denen der Therapeut ein Zugang sein könnte.

\section{Träume als Feldausdrucksformen}

Bei beiden dieser Arbeitsweisen sind Träume in erster Linie ein Ausdruck des individuellen Lebens der Klient* innen, in der Gestalttheorie ist das Selbst jedoch ein aus dem Feld entstehendes (Perls, 1978; Philippson, 2001, 2009), und dies führt zu einer Reihe anderer Möglichkeiten bei unserer Exploration von Träumen. Der «Horizont» (Husserl, 1970) all unserer Bewusstseinsakte ist unsere Mannigfaltigkeit der möglichen Bindungen in der Welt und mit anderen Menschen, von denen wir wissen, dass sie anders agieren würden: 
«The perception of a thing is perception of it within a perceptual field. And just as the individual thing in perception has meaning only through an open horizon of spossible perceptions' ... it has an external horizon> precisely as a thing within a field of things: and this points finally to the whole sworld as perceptual world ... But in living with one another each can take part in the life of the others. Thus the world exists not only for isolated men but for the community of men; and this is due to the fact that even what is straightforwardly perceptual is communalized» (ebd., S. 162f.).

Dieser Horizont ist genauso Teil der Grundlage der Träume wie unsere individuellen Angelegenheiten. Träume können also in unserer gegenwärtigen Welt Ausdruck des Gemeinschaftsfelds in der Pandemie sein, wie auch unserer individuellen Antwort darauf. Wenn das Feld, in das wir eingebunden sind, sich auf eine neue Ordnung hinbewegt (zum Beispiel eine Krise in der Familie, eine Änderung der politischen Verhältnisse in unserem Land oder eine Änderung an unserem Arbeitsplatz), dann kann das in unseren Träumen als Bilder der neuen Ausrichtung ausgedrückt werden, und diese können dann als ein voraussagender Traum gesehen werden. Er ergibt aber nur dann als eine Voraussagung Sinn, wenn ich mich selbst aus dem Feld als Beobachter*in herausnehme und nicht dessen integraler Teil oder, wie Daniel Stern (2004) es formulierte, die intersubjektive Matrix bin. Manche Kulturen haben Traditionen für voraussagendes Träumen, die in der Kultur verstandene Symbole zur Verfügung stellen, mit denen in Träumen das Ergebnis eines Gemeinschaftsprozesses geliefert werden kann.

\section{Arbeiten mit dem Traumfeld}

Betrachtet man Träume von dieser Ebene aus, bieten sich sofort andere Möglichkeiten, wie man sich ihnen in den eigenen Explorationen und in der Therapie mit Klient" innen annähern kann. Wenn ich mehrtägige Schulungen oder Therapie-Workshops abhalte, beginne ich jeden Tag nach dem ersten mit einer Einladung, die Träume aus der vorhergehenden Nacht einzubringen. Oft sind vorgetragene Träume durch die Gruppenprozesse und die Themen im Workshop geprägt und liefern neue Quellen für ihre Weiterführung. Bilder werden oft in den Träumen der Gruppenmitglieder geteilt. Wird das bemerkt, erfahren sich die Menschen neu, indem sie sich als Teil von etwas sehen, das umfassender ist als sie selbst, weil auch Bilder von Isolation und Nicht-Kommunikation mitgeteilt werden. In dieser Phase finden oft Gruppenmitglieder, die bislang still dabeisassen, auch einen Weg, sich zu äussern (ich dränge Menschen, deren Ausdrucksform das Schweigen ist, nicht zum Sprechen, aber ich äussere bewusst, dass das Schweigen, phänomenologisch gesehen, ihre Beteiligung ist und dass das konforme Sprechen, wenn man dazu drängt, weniger über ihr Wesen aussagt als über ihre Konformität).
So können wir eine kollektive Traumgeschichte entwickeln und fragen, was der nächste Schritt in der Geschichte sein wird. Oder wir können den Traum darstellen und die Personen übernehmen dabei Rollen. Bringt ein Gruppenmitglied den Traum ein, den wir betrachten, lasse ich Raum für andere, damit sie sich auf ihre Antworten auf den Traum einlassen können.

Gruppenmitglied 2: Das passt dazu, wie ich dich erlebe. Du zeigst dich immer als gross und separat und ich weiss nicht, wie wir miteinander Spass haben könnten.

Gruppenmitglied 3: Ich hatte einen ähnlichen Traum, ich fand einen Süsswarenladen, ich sah durch die Fenster, ging aber nicht hinein.

Therapeut*in: Welche Spiele könnten wir hier spielen oder wie viele Süssigkeiten teilen? Und wer ist vor der Tür? Und was hält uns zurück?

Jetzt können wir die Themen Spielen, Hemmung, Akzeptanz und Zurückweisung zusammen erforschen. Die Antwort von Gruppenmitglied 2 ist besonders interessant, weil der Stil der Antwort genau zu den Bildern passt, die von einem grossen, separaten und ernsthaften Standpunkt gleichsam zu einem niedrigeren vorgetragen werden, der sich ändern muss, wenn er dazugehören will. Der grosse Vorteil dieser Art des Arbeitens ist, dass das Thema, das jede" $r$ der Klient" innen einbringt, nicht sein"ihr individuelles Problem ist, das es zu «lösen» gilt, sondern seine*ihre Version eines Dilemmas, mit dem mehrere Personen konfrontiert sind. Die Themen gross/ klein, Akzeptanz/Zurückweisung, Spiel/Ernst, Alleinsein/ Gemeinschaft sind grösser und universeller als der* die Einzelne.

\section{Interpretation von Träumen}

Ich sagte bereits, dass ich das Konzept, bei dem Träumen oder Traumbildern Bedeutungen reduktionistisch zugeteilt werden, nicht mag. Allerdings erlaubt diese, aus dem Feld kommende Sicht auf Träume, in der Tat eine Art der Interpretation, die Gültigkeit beanspruchen kann. Auf der Ebene, auf der ein Traum Ausdruck eines mit anderen geteilten Raumes ist, kann die Bedeutung des Traums auf organische Weise allen klar werden, auch Therapeut*innen, die ebenfalls Teil des Feldes sind, und die Verbalisierung dieses gemeinsamen Verstehens kann dann eher bekräftigend als reduzierend sein. Dies ist die Ebene, auf der Schaman*innen Geschichten erzählen, um auszudrücken, was nicht in Worte gefasst, aber von der gesamten Gemeinschaft verstanden werden kann.

Ich erinnere mich, als ich einmal sehr viel zu tun und einen Traum von einem ausgebrannten Laden hatte, der mit Brettern vernagelt war, und der einzige Gegenstand, der sich ausserhalb befand, war eine Rechenmaschine, die aus dem Feuer geworfen worden war. Was ich sagen wollte, war klar und auch mein Therapeut konnte das 
als eine gemeinsame Erfahrung verstehen, und es war nicht nötig, dass wir damit weitere «Berechnungen» anstellten! Auf dieselbe Weise sind manche Träume in sich vollständig; oft sind dies Träume, bei denen man an einem neuen Ort ist, feststeckt oder sich in einem Kampf befindet, und diese Träume können auf einfache Weise mit anderen geteilt, bestätigt oder gefeiert werden.

In Bezug auf unseren Traum könnte ich auf dieser Interpretationsebene antworten:

Therapeut: Ich finde es spannend, wenn ich von Ihnen höre, dass Sie eine neue Tür finden, einen neuen Raum, neue Fenster. Das fühlt sich an, als hätten Sie einen Schritt nach vorn getan. Wissen Sie etwas mehr darüber?

Klient: Die Welt sieht für mich etwas heller aus und ich habe mit mehr Leuten gesprochen.

Therapeut: Das freut mich wirklich sehr für Sie. Ich bin sicher, dass wir hier gemeinsam neue Orte finden können.

\section{Vielschichtige Signifikanz von Träumen}

Wir können bereits sehen, dass Träume, wie jedes gute Kunstwerk, auf vielen Ebenen betrachtet werden können und wenn man sich ihnen auf unterschiedliche Art und Weise nähert, führt das zu einem anderen nächsten Moment des Prozesses. Es gibt keine «richtige» oder «falsche» Art, sich dem Traum zu nähern, bzw. könnte man sagen, die «falsche» Art wäre, eine vorgefertigte Weise zu haben, wie man mit dem Traum umgeht. Ist die Art von Therapeut*innen, wie sie mit Träumen interagieren, eingeschränkt, produzieren Träumende eingeschränkte Träume, was Ausgangspunkt für eine Kommunikation sein kann: So haben freudsche Klient*innen freudsche Träume, jungianische Klient*innen jungianische Träume.

Ich komme zurück auf den Gedanken eines bedächtigen Eintretens in den Traum, das viele Möglichkeiten für ein Sich-Einbringen eröffnet, wobei manchmal Klient*innen die Führung übernehmen, manchmal Therapeut*innen. Und dann wird das, was wir tun, Teil des «Horizonts» des Traums in der nächsten Nacht.

\section{Der Traum als Realität}

\section{Was ist real?}

«Since dreams are experienced in the relative isolation of sleep, they are usually the most original of our psychological experiences. For the typical person, in fact, the dream may be the only state where originality can become manifest ... That which seems absurd, bizarre, or meaningless in dreams only seems so from the older more established points of view and attitudes that still dominate the conscious mind»(Rossi, 1985, S. 14f.).
Im Vorwort zu seiner Arbeit mit Gloria (https://www. youtube.com/watch?v=it0j6FIxIog) spricht Fritz Perls über Gestalttherapie als «Helping the patient to wake up from the nightmare of his existence.» Ich halte das für eine bemerkenswerte Aussage, getroffen in der beiläufigen Art, in der viele von Perls tiefgründigsten Gedanken geäussert werden. Man kann das sehr salopp nehmen, wie wenn wir sagen «Der Tag heute war ein Albtraum», und dann will Perls Äusserung Klient*innen schlicht helfen, einen besseren Tag zu haben. Aber der Gedanke «the nightmare of his existence» verdient, dass man bei ihm als einer existenziellen Aussage verweilt, er öffnet den Weg zu faszinierendem Neuland. Franz Kafka (1915) schrieb über einen Mann, dessen Wirklichkeit war, dass er sich in einen Käfer verwandelt hatte, aber die Menschen um ihn herum liessen für sich diese Wirklichkeit nicht zu. War es ein Traum, dass er ein Käfer war, oder war er ein Käfer, der träumt, ein Mensch zu sein?

Führt man diese Aussage weiter, was würde es bedeuten, das problembeladene, wache Leben als einen Traum oder Albtraum zu behandeln? Und andersherum, was würde es bedeuten, die Träume einer Person als realen Ausdruck ihrer selbst zu behandeln? So bringt zum Beispiel ein sanftmütiger Klient Träume mit, in denen viel gestritten wird. Was, wenn die Realität der Person die eines Streithahnes ist und die sanftmütige Präsentation ein wiederkehrender Traum? Was würde dieser Traum bedeuten? Wie könnte ich dem Streithahn ein Therapeut sein und welche Therapie bräuchte er? Was würde der Klient durch ein Aufwachen verlieren? Zu wem würden die anderen Personen in dem Traum werden (zum Beispiel Personen, die man besänftigen oder zu denen man zuvorkommend sein muss)? Worüber muss gestritten werden?

Währenddessen gibt es in dem, was Klient*innen als ihr waches Leben sehen, eine Wiederholung bestimmter Arten von Pech, schlechten Beziehungen oder verpassten Chancen, die in der Regel Erlebnisse in ihren frühen Lebensabschnitten widerspiegeln. Das ist, was sie mittlerweile als ihr Leben erwarten, das ganz anders zu sein scheint als das Leben und die Erwartungen derer in ihrer Umgebung. Dies ist ihr Schicksal und niemand kann ihnen helfen. In der Sprache der Träume könnten wir das als immer wiederkehrenden Albtraum sehen, obwohl die Klient*innen es als Realität des Wachzustandes sehen, aus der sie nicht entkommen können - was auch das Gefühl vermittelt, sich in einem Traum zu befinden. Sie wachen mit einem Gefühl der Erleichterung auf, weil sie «nur geträumt» haben, wohl wissend, dass es in der nächsten Nacht wieder genau gleich sein wird. Genauso verlassen Klient*innen eine schmerzhafte Beziehung und fühlen Erleichterung, jedoch im Wissen, dass sie sich bald wieder im selben Albtraum befinden werden, entweder mit derselben oder einer ähnlichen Person. Behandelt man solche starren Muster als wiederkehrende Träume, versetzen wir uns in die Lage, all die Methoden der Traumarbeit einzusetzen, die ich im ersten Teil dieses Artikels genannt habe und die es zu untersuchen galt. 


\section{Leben und Träumen als Kunst}

«But the self is precisely the integrator ... It is the artist of life» (Perls et al., 1994 [1951], S. 11).

Was Leben und Träumen zusammenbringt, ist das künstlerische Schaffen. Leben und Träume sind spontane, künstlerische Schöpfungen des Selbst, das auch zutiefst feldbezogen ist. Dies ist keine Äusserung einer sozialen Konstruktion, die impliziert, dass sich das Selbst ausserhalb des sozialen Felds befinde und von ihm wie eine Art Flipperkugel beeinflusst würde, wie dies manche Schriften Kurt Lewins (2015) nahelegen. Es geht vielmehr um das Selbst als «Organ» des künstlerischen Schaffens und der Kreativität des Feldes. Für Künstler*innen gibt es keine Trennung zwischen dem Träumen und einer kreativen Handlung. Ein*e kreative Künstler*in wird niemals in die Welt "geworfen", vielmehr ist sein*ihr Eintritt in eine Situation Teil der Verfasstheit der Situation, in die er*sie eintritt. Ich bin über die Art und Weise, wie ich in einen Raum hineingehe, laut oder leise, mit gesenktem oder erhobenem Kopf und einem Lächeln oder einem verärgerten Stirnrunzeln (oder mit einem Gewehr, in einer Polizeiuniform oder mit Mopp und Eimer) Teil der Verfasstheit des Raumes, den ich entdecke. Sichergestellt wird dies durch den Aufbau unseres Nervensystems (Rizzolatti \& Craighero, 2004) und durch unsere sozialen Kompetenzen. Wir laden Menschen in unsere Wachträume ein und diejenigen, die sich uns anschliessen, sind die, die unsere Einladung annehmen. Dies ist die einzige Art, auf die unsere Leben eine Kohärenz und ein Narrativ haben. Währenddessen zeigen uns unsere Schlafträume die unvollendete Seite dieser Wachträume, sei es die Unannehmlichkeit dessen, was wir verhindert haben, oder die Freuden und Möglichkeiten, die wir aufgegeben und aus unseren Wachträumen verbannt haben.

So sind also die beiden, das Schlafen und das Wachen, nicht von Natur aus getrennt und das Problem entsteht im Akt der Trennung der beiden, sodass unsere Träume niemals auf einen Weg gelangen, auf dem sie sich in der Realität verwirklichen. Das bedeutet nicht, dass alle Träume untersucht werden müssen: Manche Träume sind nicht sonderlich interessant, genauso wie manche Erlebnisse im Wachzustand nichts zum Erforschen hergeben.

\section{Risiken des Umkehrungsprozesses}

Hier gibt es noch eine andere Seite: Menschen, die fähig sind, ihre Gemeinschaft in ihre Träume zu ziehen, sei es durch ihr Charisma oder ihre Rücksichtslosigkeit. Träume dieser Art sind Träume von landesweitem Ruhm, Träume von geheimen Feinden, die vernichtet, von minderwertigen Menschen, die unterworfen werden müssen, Träume von Dingen, die Gott einfordert. In einer homophoben Gesellschaft melden Menschen Entführungen durch Ausserirdische, von denen sie einer analen Untersuchung unterzogen werden (in dem Comedy-Science-Fiction-Film Paul sagt der Ausserirdische: «Es ist erstaunlich, wie we- nig Informationen man aus einer analen Untersuchung erhält!»). Aus dieser Perspektive könnten wir sagen, dass die Traumbilder, an denen zu arbeiten ist, Schwäche, mangelnde Bedeutung, Geheimnisse, Unterlegenheit sind: Dies sind die Traumfiguren, die in die Therapie aufzunehmen sind.

\section{Anwendung auf die Paartherapie}

Ich stelle immer wieder fest, dass diese Art des Denkens bei der Arbeit mit Paaren, die ständig im Streit liegen, nützlich sein kann. Wenn die Beziehung zum*zur Partner*in ein sich laufend wiederholender Wachtraum ist, auf den die Methoden der Traumarbeit angewendet werden können, sind die Auseinandersetzungen des Paares ein wesentlicher Teil dieses Traumprozesses. Ich bitte das Paar daher, den Streit fortzusetzen, aber dabei genau die gegensätzliche Position einzunehmen. Ich habe nie erlebt, dass Paare damit Schwierigkeiten haben. Ich erkläre das damit, dass beide Partner*innen beide Positionen in sich haben, aber dass sie genauso, wie sie entscheiden, dass der*die eine kocht und der*die andere den Abwasch macht, sich so organisieren, dass der*die eine Partner*in die eine Position einnimmt (wobei es oft darum geht, keine zu grosse Nähe aufkommen zu lassen oder zu viel kontrolliert zu werden) und der*die andere die gegensätzliche Position (sich zu weit zu entfernen und zu unorganisiert zu werden). Die Traumarbeit ermöglicht beiden Partner*innen, die beiden Positionen zu identifizieren.

\section{Arbeiten mit der Welt als gelebtem Traum}

Ich habe festgestellt, dass Klient*innen von dieser Herangehensweise fasziniert und sehr bereit sind, sie auszuprobieren. Dies hat zu einigen sehr spannenden Explorationen geführt, bei der alle Arten der Traumarbeit mit der Beschreibung ihrer jeweiligen Leben eingesetzt werden können (zum Beispiel ihre Beziehung oder wie sie die Menschen um sich herum sehen). Ein Beispiel:

Klient: Ich schreibe an einem Buch, und das schon seit Jahren, aber ich verzettele mich ständig und kann es nicht zu Ende bringen.

Therapeut: Kannst du experimentieren und dasselbe als Buch sagen?

Klient: Ich bin unvollständig. Ich habe einiges Gutes in mir, aber wenn er nicht fertig wird, werde ich niemals das Licht der Welt erblicken und niemand wird mich zu Gesicht bekommen.

Therapeut: Was sagst du also zu deinem Autor?

Klient: Lass mich raus und lass mich los! Ich bin gut genug, um der Welt gegenüber zu treten.

Therapeut: Und jetzt sei der Autor.

Klient: Ich weiss nicht, ob du gut genug bist. Vielleicht lachen die Leute, wenn ich dich gehen lasse, und sagen, dass es ein schlechtes Buch ist. 
Therapeut: Und jetzt sei die Leute, die lachen und den Autor und das Buch kritisieren.

Klient: Was soll denn das für ein Roman sein? Du denkst nur, du kannst ein Buch schreiben, aber du kannst es nicht.

Therapeut: [in der Traumarbeit ist es wichtig, zu sehen, wo die Energie ist; jetzt haben wir eine engagierte und energetische Figur] Wie fühlt sich das an, das dem Autor zu sagen? Du scheinst ja eine Menge Energie zu haben. Was ist dir so wichtig?

Klient: Ich weiss nicht - ich mag einfach keine schlechten Bücher.

Therapeut: Bleib dran, hier scheint etwas viel wichtiger zu sein als das.

Klient: [eine Zeitlang Schweigen, dann Tränen] Was immer ich gemacht habe, für meinen Vater war es nie gut genug. Er hat mich angeschrien, dass ich es schlecht gemacht habe, er schien so stark und ich war so schwach und hilflos. Ich empfand es immer als stärker, etwas zu kritisieren als etwas zu machen. Das ist schlecht, oder?

Therapeut: Ich konnte nicht in die Position des Kritikers versetzt werden. Ich verstehe, was du meinst. Auf eine gewisse Weise ist das eine perfekte Kurzgeschichte. Ist das die, die du weiterschreiben willst?

Klient: [sieht verwundert aus] Das Buch, das ich schreibe, ist über jemanden, der denkt, er sei toll, der es aber nicht ist.

Der Therapeut überlässt es dann dem Klienten, ob er weitermachen will. Dieser entscheidet dann, dass er das Buch weiterschreibt, aber auf eine ganz andere Art und Weise.

\section{Gelebte Umgebung als Teil des Traums}

Bei Perls sind in der Traumarbeit nicht nur lebende Wesen wichtige Figuren im Traum. Orte und Gegenstände tragen ebenfalls zum Hintergrund bei, formen die Szenerie mit und geben ihr eine Klangfarbe. Genauso sind Gegenstände, mit denen sich eine Person umgibt, wichtige Bestandteile des Fortbestehens des Wachtraums, den sie lebt. Damit sich ein Traum ändert, müssen Klient*innen den "container» des Traums ändern. Depressive Klient*innen, die aus der Depression herauskommen, müssen in der Regel ändern, was sie sehen, wenn sie das erste Mal aufwachen, was für gewöhnlich eine Reflexion ihrer Depression ist (oder ihre Depression ist eine Reflexion ihrer Umgebung!). Sie müssen die Wände in einer anderen Farbe streichen, neue Gegenstände anschaffen, mit denen sie eine Verbindung herstellen können, andere Kleidung tragen. Bisweilen geschieht dies spontan, manchmal kann das in Form eines Experiments vorgenommen werden. Sie werden auch feststellen, dass sie anders mit den Menschen in Verbindung treten und manchmal mit anderen Menschen. Das ist wie bei der Traumarbeitsmethode, in der Menschen den Traum bewusst ändern und ein neues Ende finden.

\section{Fazit}

Ich habe meine Sichtweise auf das Träumen und die Art und Weise, wie man mit Träumen arbeiten kann, dargelegt. Träume als Kunstwerke zu betrachten, erscheint mir wichtig, weil so zum einen eine bedächtigere und weniger kognitive Annäherung an Träume und Träumende möglich wird, und zum anderen kann man sehen, auf welche Weise Traumprozesse integraler Teil der Kunstfertigkeit sind, mit der wir unsere Leben leben, sei es im Schlaf oder im Wachzustand.

\section{Literatur}

Husserl, E. (1970). The Crisis of European Sciences and Transcendental Phenomenology. Evanston/IL: Northwestern UP.

Kafka, F. (1915). Die Verwandlung. Die Weißen Blätter, 2(10), $1177-1230$

Lewin, K. (2015). Principles of Topological Psychology. Eastford/CT: Martino Fine Books.

Perls, F. (1978) Finding Self through Gestalt Therapy. Gestalt Journal, I(1), 54-73.

Perls, F., Hefferline, R. \& Goodman, P. (1994 [1951]) Gestalt Therapy: Excitement and Growth in the Human Personality. New York: Gestalt Journal Press.

Philippson, P. A. (2001). Self in Relation. Highland/NY: Gestalt Journal Press.

Philippson, P.A. (2009). The Emergent Self: An Existential-Gestalt Approach. London: Routledge.

Rizzolatti, G. \& Craighero, L. (2004). The Mirror-Neuron System. Annual Review of Neuroscience, 27, 169-192.

Rossi, E. L. (1985). Dreams \& the Growth of Personality. New York: Brunner/Mazel.

Stern, D. N. (2004). The Present Moment in Psychotherapy and Everyday Life. New York: Norton.

Zeigarnik, B. (1927). Über das Behalten von erledigten und unerledigten Handlungen. Psychologische Forschung, 9, 1-85.

\section{Rêver dans le monde}

Résumé : À mon avis, les rêves sont très importants à bien des égards. Je vois ici une signification similaire à celle de Sigmund Freud et de Fritz Perls. J'intègre régulièrement le travail sur les rêves dans mes thérapies, mes formations et mes travaux de groupe afin que notre perception soit orientée vers ce qui se passe au niveau individuel et collectif. Mon article est divisé en deux parties. La première partie est un aperçu de la façon dont je vois les rêves et des différentes façons dont j'ai travaillé sur eux en tant que thérapeute. Dans la deuxième partie, je présente une méthode de travail sur les rêves que j’ai développée au cours des dernières années. La compréhension et le travail prennent alors une orientation différente, où la séparation stricte entre l'état de veille et le rêve est supprimée.

Mots clés : rêve, œuvres d'art, processus de terrain, interprétation, rêves éveillés, rêves comme réalité, couples

\section{Sognare nel mondo}

Riassunto: Secondo la mia opinione, i sogni sono importanti per svariati aspetti. A questo proposito un significato simile veniva 
loro dato da Sigmund Freud e Fritz Perls. Sono solito inserire il mio lavoro sui sogni nelle sedute di terapia con i pazienti, nelle formazioni e nei gruppi di lavoro affinché la nostra percezione sia orientata a cogliere ciò che accade sia sul piano individuale che collettivo. Il mio articolo si divide in due parti. La prima è costituita da una panoramica sulla definizione di sogno da me fornita e sulle modalità del lavoro svolto con il materiale onirico in qualità di terapeuta. Nella seconda parte, presento una metodologia di lavoro con i sogni che ho sviluppato negli ultimi anni. A questo proposito sia la comprensione che il lavoro vengono spinti verso una direzione in cui la rigida divisione tra lo stato di veglia e l'attività onirica si fa via via più labile.

Parole chiave: sogno, opere d'arte, processi del campo, interpretazione, sogni in stato di veglia, sogni come realtà, coppie

\section{Der Autor}

Peter Philippson, M.Sc., ist ein bei der UKCP eingetragener Gestalttherapeut sowie Ausbilder, Lehrkraft und Supervisor beim Gestalt Psychotherapy \& Training Institute UK. Er ist Gründungsmitglied des Manchester Gestalt Centre, Vollmitglied des New York Institute for Gestalt Therapy, Führender Ausbilder für GITA (Slowenien), Fakultätsmitglied der IPsiG (Turin), Beiratsmitglied des Center for Somatic Studies, Gründungsmitglied von IG-FEST und ehemaliger Präsident der International Association for the Advancement of Gestalt Therapy.

\section{Kontakt}

Peter Philippson

Manchester Gestalt Centre

7 Norman Road

Manchester, M14 5LF, UK

E-Mail: peterphilippson@gmail.com

Website: www.mgc.org.uk 